\section{Skeletal and cranio-facial signs in Gorlin syndrome from ancient Egypt to the modern age: sphenoid asymmetry in a patient with a novel PTCH1 mutation}

\author{
Giovanni Ponti ${ }^{*}$, , Cristel Ruini ${ }^{2}$, Lorenza Pastorino ${ }^{3}$, Pietro Loschi ${ }^{4}$, Annarita Pecchi ${ }^{5}$, \\ Marcella Malagoli ${ }^{5}$, Victor Desmond Mandel ${ }^{2}$, Rosa Boano ${ }^{6}$, Andrea Conti², \\ Giovanni Pellacani² \& Aldo Tomasi ${ }^{1}$
}

\begin{abstract}
Gorlin syndrome is an autosomal dominant disorder linked to PTCH1 mutation, identified by a collection of clinical and radiologic signs. We describe the case of a family in which father and son fulfilled clear cut diagnostic criteria for Gorlin syndrome including multiple basal cell carcinomas, keratocystic odontogenic tumors, atypical skeletal anomalies and a novel PTCH1 germline mutation (c.1041delAA). Craniofacial and other skeletal anomalies displayed at 3D and helical CT scan were: macrocephaly, positional plagiocephaly, skull base and sphenoid asymmetry, bifidity of multiple ribs and giant multilocular odontogenic jaw cysts. Extensive multilamellar calcifications were found in falx cerebri, tentorium, falx cerebelli and in the atlanto-occipital ligament. The inclusion of bifid ribs as a novel major criteri may be useful for the recognition and characterization of misdiagnosed cases.
\end{abstract}

Gorlin-Goltz or nevoid basal cell carcinoma syndrome (NBCCS) is an autosomic dominantly inherited disorder associated to $P T C H 1$ germline mutations, which is involved in the proliferative $S H H$ pathway [1,2]. The phenotypic expression of the disease includes a wide spectrum of skeletal anomalies, congenital malformations and the predisposition to the development of various neoplasms, such as multiple basal cell carcinomas (BCCs), keratocystic odontogenic tumors (KCOTs), ameloblastomas, ovaric and cardiac fibromas and medulloblastomas $[3,4]$. Skeletal anomalies are extremely various in patients with NBCCS and affect both axil and appendicular components. Bifid ribs, macrocephaly, frontal bossing, skull base asymmetry, syndactyly or polydactyly, pectus excavatum and carinatum, vertebral anomalies are among the most represented [5].

PTCH1 mutation carriers can also be affected by hypogonadotrophic hypogonadism (5-10\%), cryptorchidism or gynecomastia, but most individuals are able to reproduce. This is well demonstrated by the survival of the genetic mutation throughout the centuries, since peculiar skeletal anomalies characteristic of NBCCS (i.e., jaw KCOTs, bifid ribs, frontal bossing) were also found in ancient Egyptian mummified individuals belonging to archaeological collections that we have personally documented and further characterized [6].

NBCCS is a complex entity and its evaluation is based on different criteria (Box 1) [4]. Since Kimonis et al. described major and minor criteria, other diagnostic criteria have been proposed

'Department of Diagnostic \& Clinical Medicine \& Public Health, University of Modena \& Reggio Emilia, Modena, Italy

2Department of Dermatology, University of Modena \& Reggio Emilia, Modena, Italy

${ }^{3}$ Department of Internal Medicine \& Medical Specialties (DiMI), University of Genoa, Genoa, Italy

${ }^{4}$ Department of Plastic \& Reconstructive Surgery, University of Modena \& Reggio Emilia, Modena, Italy

${ }^{5}$ Department of Radiology, University of Modena \& Reggio Emilia, Modena, Italy

${ }^{6}$ Department of Life Sciences \& Systems Biology, University of Turin, Turin, Italy

*Author for correspondence: Tel.: +39 059 4222600; Fax: +39 059 4222647; giovanni.ponti@unimore.it

\section{Future ONCOLOGY}

.

.

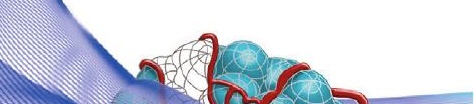

\section{KEYWORDS}

- bifid ribs • falx cerebri calcifications • Gorlin-Goltz syndrome $\bullet$ nevoid basal cell carcinoma syndrome - PTCH1 • sphenoid anomalies • volume CT scan 
Box 1. Major and minor criteria for the diagnosis of nevoid basal cell carcinoma syndrome.

\begin{tabular}{l} 
Major criteria \\
- Multiple (>two) basal cell carcinomas at any age or one basal cell carcinoma less than 30 years or more \\
- Histologically proven odontogenic keratocyst or a polyostotic bone cyst \\
- Palmar or plantar pits (three or more) \\
- Ectopic calcification: lamellar or early (<20 years) calcification of the falx cerebri \\
- Family history of Gorlin's syndrome \\
Minor criteria \\
- Congenital skeletal defects: bifid, fused, splayed or missing rib, or bifid, wedged or fused vertebra \\
- Large head with occipitofrontal circumference > 97 th percentile, with frontal bossing \\
- Cardiac or ovarian fibroma (benign tumor in heart or ovary) \\
- Medulloblastoma (a malignant brain tumor that usually arises in young children) \\
- Lymphomesenteric cysts (abdominal cysts full of lymph fluid) \\
- Congenital malformation: cleft lip and/or palate, polydactyly (extra fingers or toes), congenital eye \\
defect such as cataract, microphthalmos (small eye) or coloboma (iris tumor) \\
\hline Data taken from [4].
\end{tabular}

and vary by source, but few studies have tried to define the sensitivity and specificity of which phenotypic combination is most accurate for diagnosis. Diagnostic criteria should therefore be considered as dynamic entities and should be widened for the earlier and most accurate diagnosis of syndromic settings, especially after the widespread of novel and extremely accurate diagnostic techniques, such as volume and 3D CT scan. We describe here a family displaying a clear cut full phenotype of NBCCS, highlighting the potential diagnostic value of a peculiar sphenoid dimorphism among cranio-facial anomalies, detected at a deepened volume CT scan study. Moreover, particular attention was given to NBCCSrelated skeletal anomalies, found in mummified Egyptian skeletons belonging to the osteologic collection of the Anthropology Institute of Turin (Turin, Italy).

\section{Material \& methods \\ - Patients}

The NBCCS family was examined in the department of Dermatology of the University of Modena and Reggio Emilia (Modena, Italy). Family and personal history was accurately collected through interviews and clinical charts. The NBCCS diagnostic criteria that we used were those updated by Kimonis et al. in 1997 [4], and detailed by Evans and Farndon in 2002 [7]. The diagnosis of NBCCS was established when two major or one major and two minor criteria were present. Blood samples were acquired after specific informed consent. This study was approved by the Ethics Committee of the University Hospital of Modena.

\section{- PCR amplification \& RNA isolation}

Genetic analysis was performed as was previously described by our group [8].

\section{Results}

Grandfather, father and son were brought to our attention owing to the presence of multiple BCCs and further investigated for other signs of cancer hereditary setting (Figure 1). The first family member to be examined was EB, born 1984 , who referred to our clinic at the age of 10 years because of multiple skin lesions of the back and abdomen. At the age of 13 years, orthopantomography showed well defined multiple multilocular radiolucencies with sclerotic borders, $15 \mathrm{~cm}$ in diameter, in the left and right emi-mandibular body. Maxillar bone presented one lesion in the right part $(2.3 \mathrm{~cm})$ and one in the left $(2 \mathrm{~cm})$ part. All of them were histologically examined and resulted KCOTs. KCOTs were associated to unerupted lower second and third molars and upper right third molar and central incisor. Roots of lower left first and second molars were also dislocated by KCOTs. The father, DB, born 1948, presented with frontal bossing and multiple BCCs of the back, chest and front. In 1999, resection of the left mandible branch and reconstruction with a free vascularized fibular graft was performed because of a giant multilocular histologically proven KCOTs (Figure 2).

Both patients developed further superficial and nodular BCCs over the years (Figure 1). EB 
also presented with scoliosis of the nasal septum, deviation of the anterior wall of the sella turcica and calcification of the falx cerebri. The total body CT scan highlighted pectus excavatum, bifidity of third, fourth, sixth and seventh right rib and second, fourth and fifth left rib, scoliosis of dorsolumbar spine, deformation of seventh cervical, first and second thoracic vertebrae (butterfly-like), schisis of the posterior arch of the seventh cervical vertebra and of the first sacral vertebra (Figure 3). Other characteristic findings of NBCCS were true gynecomastia, renal cysts, accessory spleen and a mild cognitive impairment. He is now awaiting for the surgical removal of recurrent KCOTs of the maxillary bone.

Skull radiographs, helical and volume CT scan showed macrocephaly and skull base asymmetry (positional plagiocephaly), mandibular prognathism (skeletal Class III malocclusion), mandibular condylar deformation and hyperplasia of the coronoid process. Giant multilocular cysts, probably corresponding to KCOTs, were located in the maxilla, left mandibular body and ramus (Figure 2). Extensive multilamellar calcifications were found in falx cerebri and tentorium cerebelli; moreover, we found small calcifications of in the apical ligament and atlanto-occipital membrane (Figure 2). A helical CT scan showed asymmetry of both sphenoid wings with thickening of the left wing, together with irregularity of trabecular bone architecture with alternating osteolytic and sclerotic areas (Figure 4).

In addition, the proband's grandfather had undergone surgery for excision of multiple BCCs. Genetic analysis of peripheral blood samples revealed the presence of a novel PTCH1 germline mutation (c.1041delAA), that has not been described so far in other families.

\section{Discussion}

A deepened radiological examination using a 3D and volume CT scan allowed us to characterize, in detail, the peculiar cranio-facial anomalies that can be found in the NBCCS hereditary syndromes that can support the diagnosis of complex or misdiagnosed cases of NBCCS. In particular, we highlighted the potential diagnostic role of sphenoid anomalies and bifid ribs among the other craniofacial and skeletal aberrations.

Abnormalities of the sphenoid bone are not very common, and consist of differently

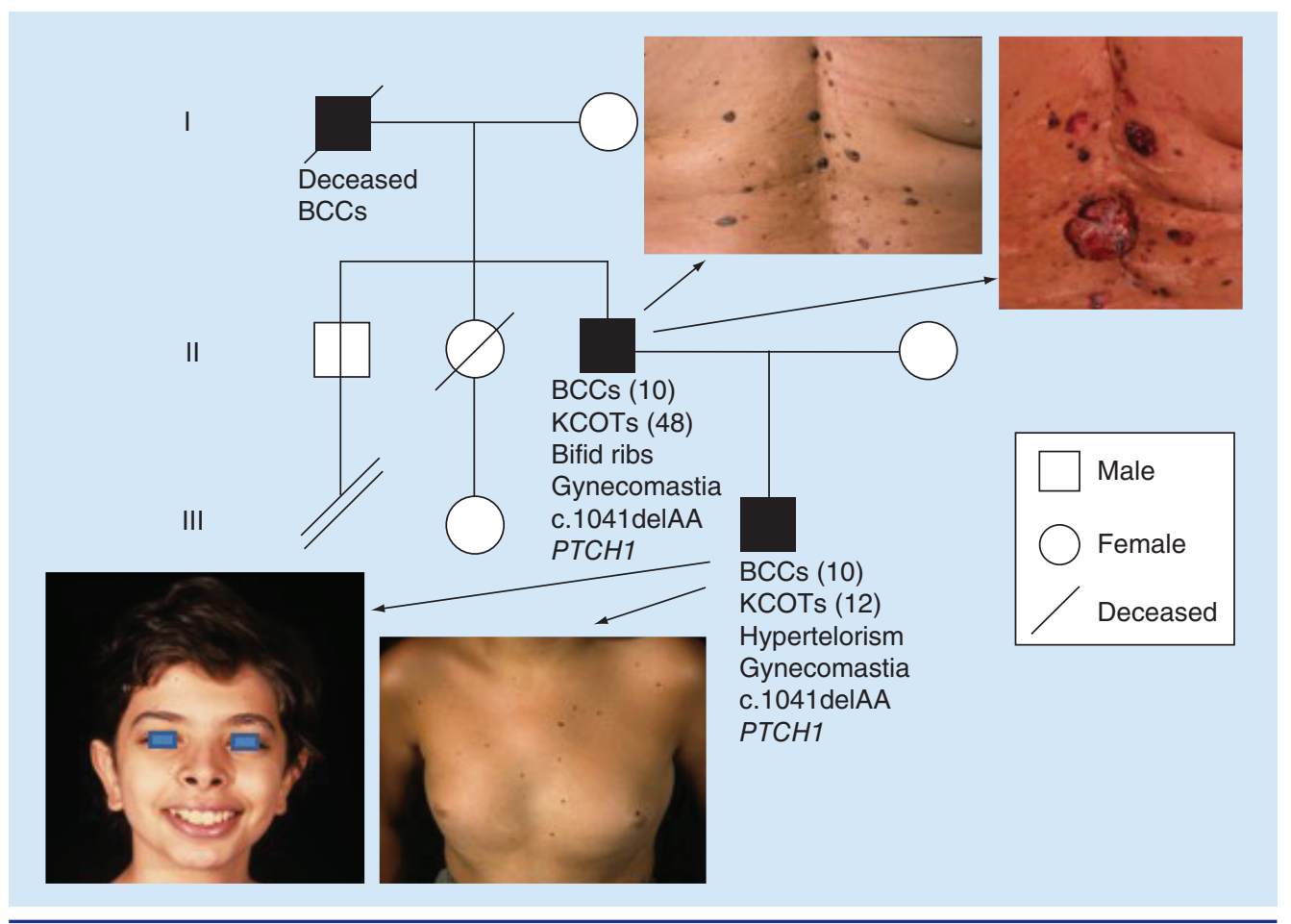

Figure 1. Family tree and clinical features of the patients with nevoid basal cell carcinoma syndrome gene carrier of the PTCH1 mutation c.1041delAA.

BCC: Basal cell carcinoma; KCOT: Keratocystic odontogenic tumor. 

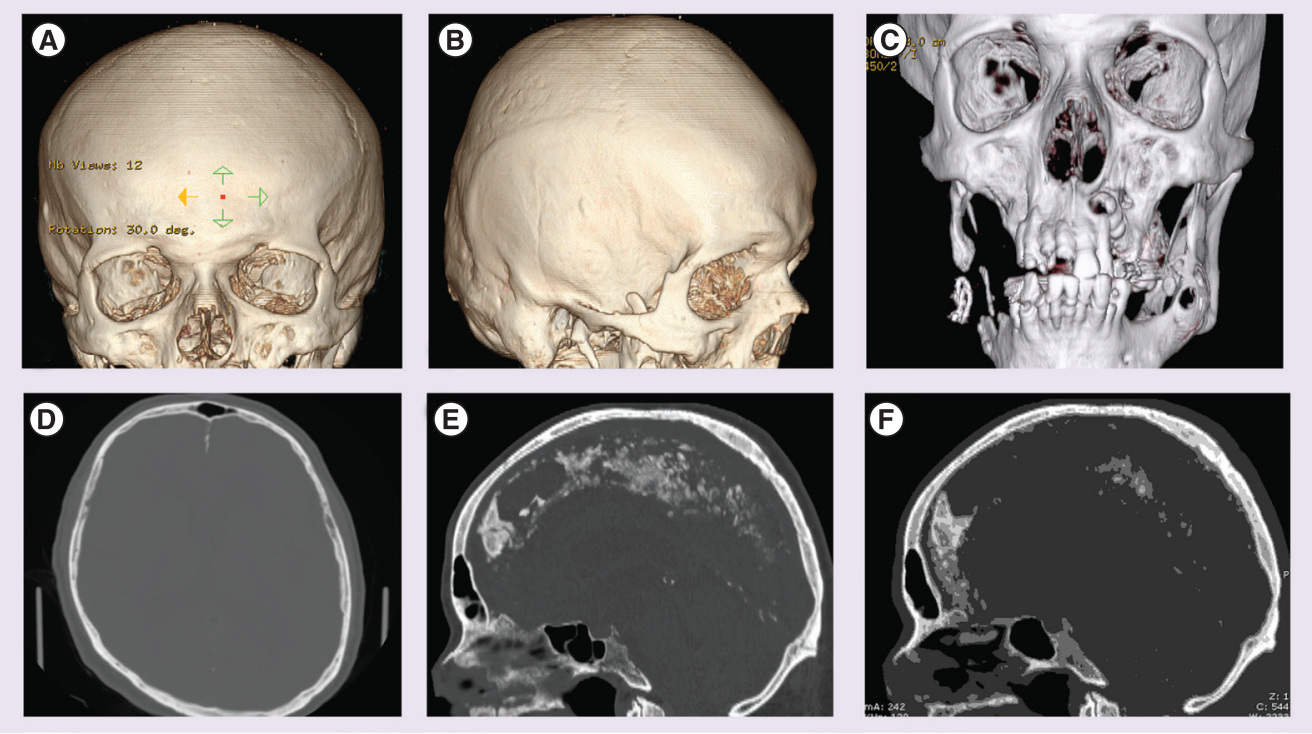

Figure 2. Radiologic study of cranio-facial anomalies in the 65-year-old proband with nevoid basal cell carcinoma syndrome. (A) Frontal view of the skull 3D CT scan. (B) Lateral view of the skull 3D CT scan of skull base shows asymmetry and frontal bossing (minor diagnostic criteria). (C) Frontal view of the skull 3D CT scan; detail: keratocystic odontogenic tumor of the left mandibular body and ramus and left maxilla, a stigmata of Gorlin syndrome (major diagnostic criterion). The patient had undergone surgery and bone craft reconstruction of the right mandibular body and ramus for keratocystic odontogenis tumor. (D) Detail of the skull: mild asymmetry of the right parietal-occipital calvarial vault (positional plagiocephaly). (E) CT scan highlights extensive multilamellar calcifications of falx cerebri, tentorium cerebelli and apical segment of the atlanto-occipital ligament. (F) Axial CT scan; detail: multilamellar calcifications of falx cerebri (major diagnostic criterion).

aggressive entities: some of them are typical of the pediatric age. Critical skull base structures can be affected, due to the central location of the sphenoid bone and an adequate preoperative imaging is mandatory for a correct diagnosis and proper surgical planning in these patients.

Sphenoid bone and in general skull base is often affected by fibrous dysplasia, an idiopathic skeletal developmental anomaly occurring in children, where normal bone is replaced with fibrous bone tissue. When the diseases is polyostotic and associated to $c a f e$-au-lait spots and endocrine diseases, such as precocious puberty, a diagnosis of McCune-Albright syndrome has to be suspected (mosaicism of GNAS1). Fibrous dysplasia of the sphenoid bone has been seen in other genetic syndromes such as Keipert or Nasodigitoacoustic syndrome (X-linked recessive) [9] and neurofibromatosis type 1 associated with plexiform neurofibroma. Fibrous dysplasia of the extremity was also misdiagnosed in a patient successively diagnosed with NBCCS [10], while the coexistence of fibrous dysplasia of maxilla, temporal bone and sphenoid sinus and unicystic ameloblastoma in a patient with no other signs for NBCCS has been reported $[11,12]$. To our knowledge, no peculiar sphenoid anomalies in Gorlin-Goltz patients have been described so far, except for bridging of the sella turcica and hyperpneumatization of sphenoid sinus [5,13].

It is thanks to volume CT scan that we could accurately investigate the intracranial anomalies highlighting the marked asymmetry of the sphenoid wings (Figures $2 \& 4$ ). In our opinion, the synchronous diagnosis of these anomalies together with other characteristic cranio-facial signs of early onset may allow the early diagnosis of NBCCS even in selected pediatric patients before the full phenotype is expressed. Nevertheless, the presence of concomitant asymmetric macrocephaly and other radiologic findings. Sphenoid asymmetry and intracranial and soft-tissue calcifications should be a critical diagnostic aid for this hereditary disorder. At this regard, the calcification of the atlantooccipital ligament has been recently described 
by Lo Muzio et al. [14] as one suitable criteria for the diagnosis of Gorlin syndrome (GS) [10]. However, we cannot consider the peculiar finding of calcification of the apical ligament detected in our patient as specific for this syndromic setting because the above mentioned study evaluated only radiograms, which do not allow an accurate study of the anatomic relationships. A $3 \mathrm{D}$ scan can allow a better characterization and can be used in future studies on wider case series that can provide a more accurate description of pathognomonic calcifications in GS.

In this patient, a volume CT scan showed asymmetry in both sphenoid wings with thickening of the left wing, irregularity of the architecture of trabecular bone with alternated osteolytic and fibrous areas. Lacking the histopathologic examination, we cannot infer that the above mentioned alterations are congenital and linked to true bone dysplasia; the altered architecture may be considered the consequence of dimorphisms due to a defect in the regeneration of trabecular bone. These findings cannot be attributable to Paget disease, fibrous dysplasia or other bone dysplastic syndromes, but may be peculiar for skeletal anomalies in GS, linked to the altered pathways in the development of mesenchymal structures.

Falx cerebri calcification can be an incidental radiologic finding and physiological variant, but is often associated to hereditary syndromes. In particular, it is found in $65 \%$ of patients with GS [5], but also Papillon-Lefèvre (palmoplantar keratoderma with periodontitis) and Hallermann-Streiff syndrome (oculomandibulodyscephaly syndrome), craniofacial dystostosis with diaphyseal hyperplasia. Moreover, infectious (i.e., neurocysticercosis), metabolic diseases (hypervitaminosis D) and tumors (chondromas) can be suspected. In the context of GS calcifications of the falx cerebri are usually multilamellar, disposed in multiple parallel strands at a respective distance of $0.52 \mathrm{~mm}$; this particular subtype has been described has type IV group of falx calcifications and is considered as specific for GS in a retrospective study on 4787 radiograms. The remaining three described groups were characterized by calcifications that differed significantly
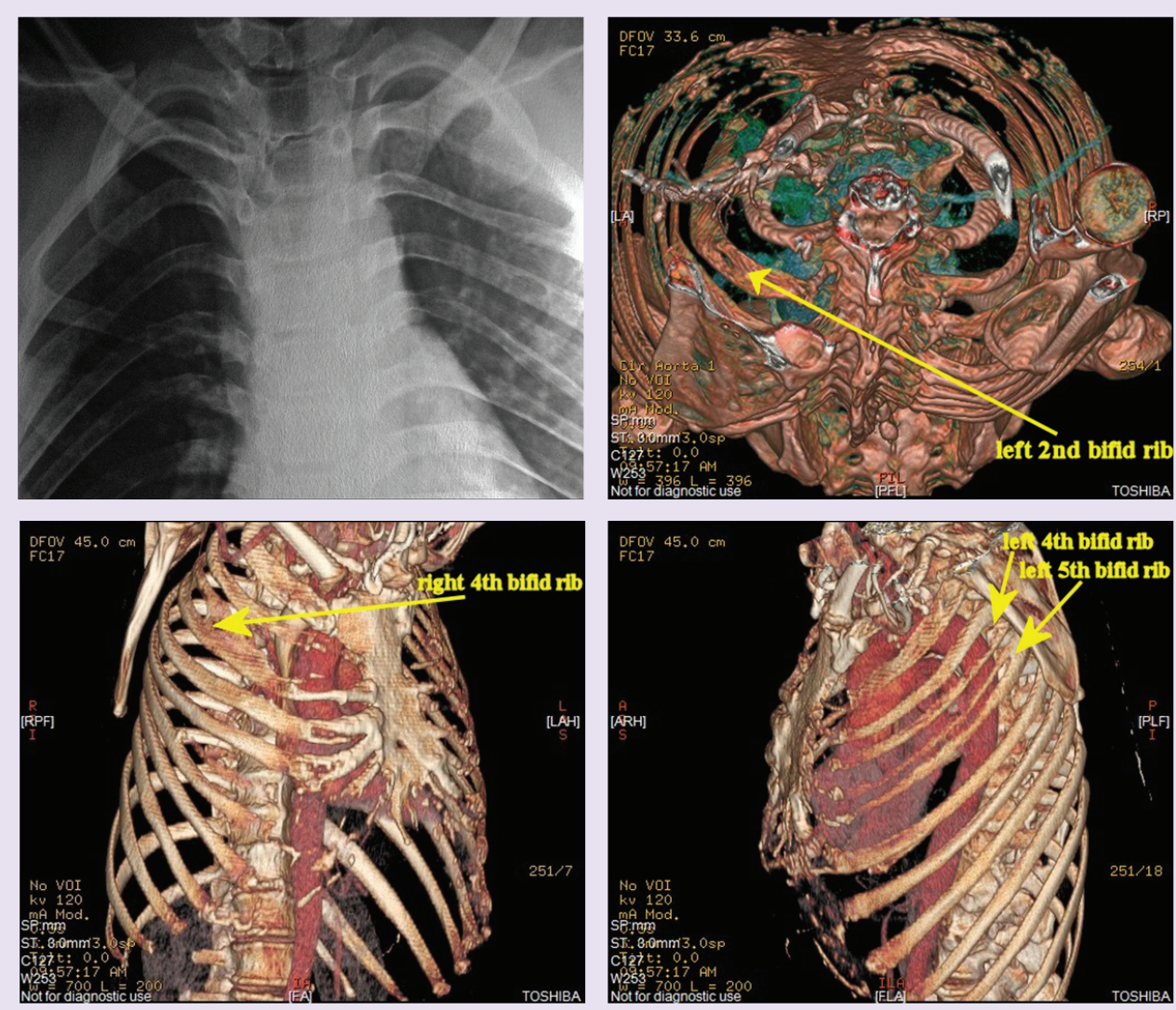

Figure 3. A 65-year-old man with Gorlin-Goltz syndrome. Radiologic findings (x-rays and 3D CT scan) of the proband with highlighted bifid ribs (arrows) are demonstrated. 
in form and extent from this particular subtype, appearing as line-shaped shading (type 1), divergent shading (type 2) and patchy and diffuse shading (type 3) [15]. Falx calcifications are part of the major criteria for the diagnosis of the syndrome. The main malformative rib lesions are bifid ribs, rib spurs and widened ribs. Bifid ribs occur when the sternal end of a rib is cleaved into upper and lower divisions. Each part has its own costal cartilage, that may fuse before articulating with the sternum. Their prevalence in the general population is reported approximately $1.4 \%$, and is higher in males. Bifidity usually involves the anterior fourth and third rib, followed by the fifth, sixth and second ribs and can be an isolated finding, as well as part of genetic syndromes or associations of malformations. Hereditary settings include NBCCS (PTCH1), Robinow syndrome (ROR2), Seckel syndrome $(A T R)$, Jarcho-Levin syndrome (DLL3), cerebro-costo-mandibular syndrome, spondylocostal and spondylothoracic dysostoses. Nonrandom associations of birth defects that comprise bifid ribs are vertebral anomalies, anal atresia, cardiovascular anomalies, tracheoesophageal fistula, renal and/or radial anomalies and limb defects; and Müllerian duct aplasia, renal aplasia, and cervico-thoracic somite dysplasia [16]. A single bifid rib is most commonly a normal incidental finding and may be detected as a palpable chest wall mass; it is usually considered a normal anatomic variant and it's often asymptomatic. However, bifid ribs, especially when bilateral, may cause musculoskeletal pain or intercostal nerve entrapment. Differential diagnosis of bifid ribs are infections (osteomyelitis), tumors (Ewing sarcoma and chondroma) and trauma. Bifid ribs are also found in approximately $26 \%$ of patients with NBCCS $[5,14,17,18]$. The occurrence of bifid ribs is quite rare in the general population, with an estimated prevalence of $0.15-3.4 \%$, increasing to $26 \%$ in patients with NBCCS [5]. The association of rib and other skeletal anomalies with tumors, especially KCOTs, is even less frequent, so that the combination of these findings has become part of the diagnostic criteria for NBCCS. This rare association was noted in 1967 by Wells and Satinoff in two mummified skeletons of the Egyptian osteological collection of the Turin Antropology Institute; the former, in fact, suggested to "look for bifid ribs" of the same body where dentigerous cysts were found as "it would be a splendid find if he discovered the dual anomaly!". In this way, the two archeologists diagnosed NBCCS (named dentigerous cyst-bifid ribs syndrome) in two skeletons excavated at Assiut (Egypt) approximately 4000 years old (Figure 5) [6]. The finding of the peculiar phenotype of GS in mummies of Egyptian dynasties demonstrates the relative benignity of NBCCS that allows, in most cases, the survival of the affected individual to the fertile age, so that the
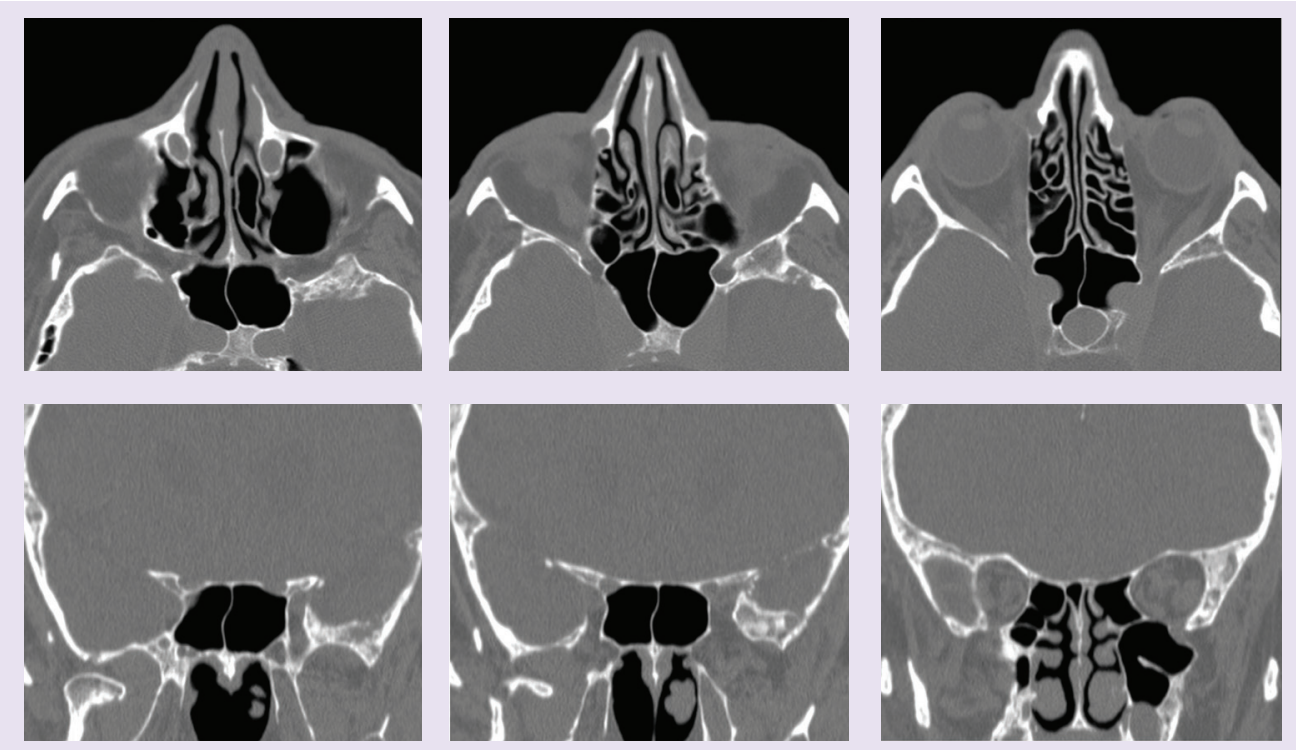

Figure 4. Sphenoid anomalies in a 65-year-old man with Gorlin-Goltz syndrome (same patient as Figure 3). A multiplanar CT scan reconstruction confirms left sphenoid wings thickening and irregularity of the trabecular bone's pattern, with alternating osteolytic and osteosclerotic areas. 

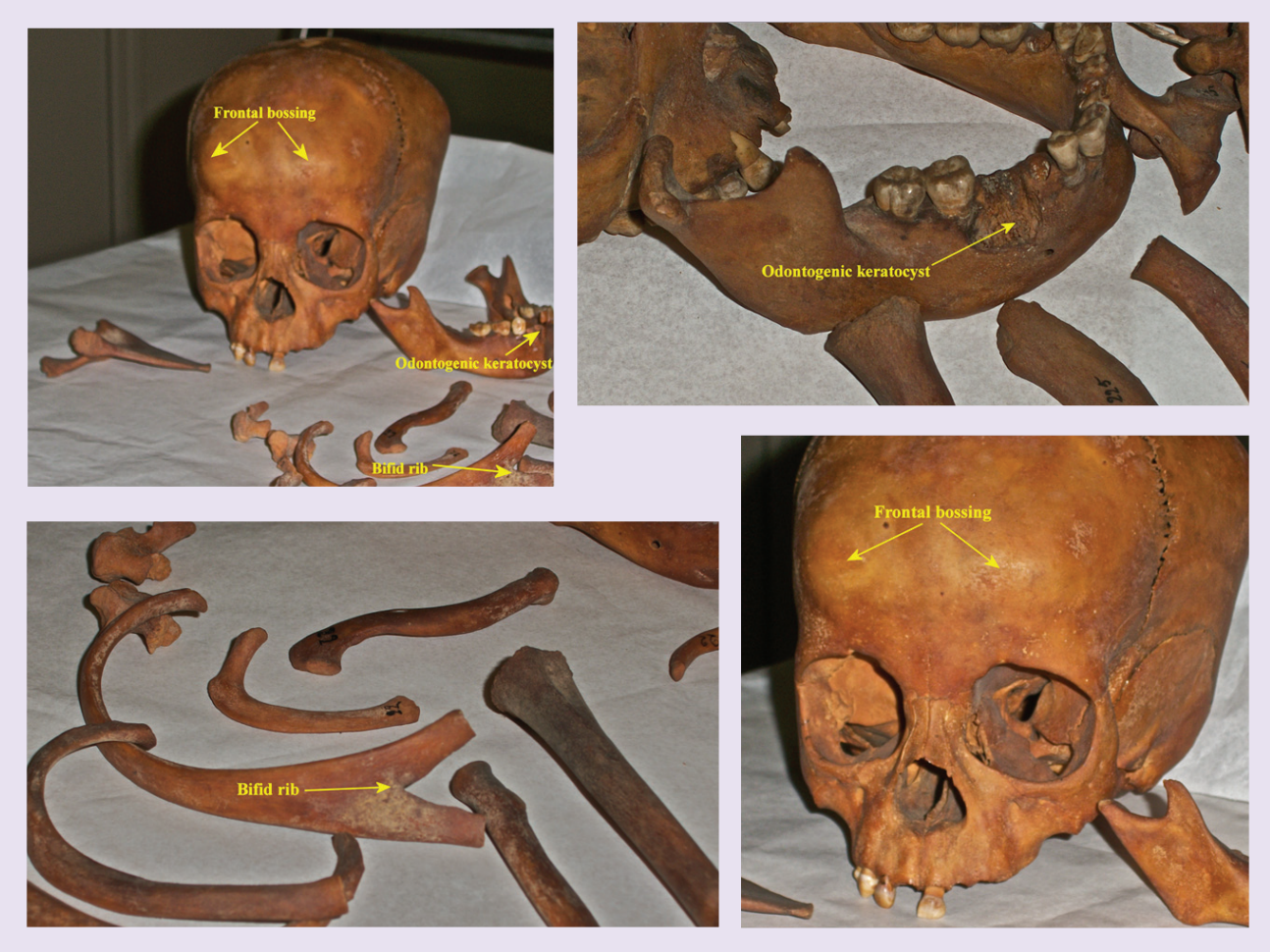

Figure 5. Skeletal anomalies characteristic of nevoid basal cell carcinoma syndrome found in two Egyptian skeletons, belonging to the anthropological museum of the University of Turin (Turin, Italy). Frontal bossing, odontogenic keratocystic tumors, bifid ribs (arrows).

Courtesy of Emma Rabino-Massa.

germline PTCH1 mutation has been preserved throughout millenniums of evolution.

Bifid ribs and other skeletal abnormalities represent a peculiar hallmark of NBCCS, together with a wide spectrum of signs and symptoms [19]. The reason why rib defects are easily found in association with other malformations has to be identified in the embryologic development: ribs develop from the costal process of the primordial thoracic vertebrae through endochondral ossification; those structures share the same mesodermal origin as cardiac muscle, kidney and skin and can, therefore, be affected by multiple malformations.

\section{Conclusion}

The collection of anamnestic data and the clinical screening for BCCs and KCOTs represent a basic stage in the NBCCS diagnosis and for the clinical differentiation from other syndromes that can share similar skeletal anomalies [20]. Although the finding of a single bifid rib, sphenoid anomalies or intracranial calcifications can be recognized as a normal anatomic variant, the synchronous presence of these aberrations in the general population is so low that it deserves a deepened clinical examination at least through a complete and accurate familial and personal history. At this regard, a 3D and volume CT scan can be an effective tool in the diagnosis and characterization of early-onset cranio-facial aberrations and other skeletal anomalies, since it allows the detection of details that wouldn't be visible with traditional radiograms and it enables a deepened anatomical study together with a preoperative assessment.

\section{Future perspective}

The NBCCS diagnostic criteria, reviewed by Kimonis, found their confirmations in the successive genetic, biomolecular and epidemiological studies [4]. However, they should be considered dynamic entities that can be improved and better characterized. Thus, the application of new criteria (i.e., peculiar calcifications of ligaments, sphenoid asymmetry and/or bifid rib as major NBCCS criterion) to a wider case series can lead to the early diagnosis of GS especially in pediatric patients, when the full phenotype is not yet expressed. 
Acknowledgements

The authors gratefully acknowledge Professor E Rabbino Massa (Department of Life Sciences and Systems Biology, University of Turin, Turin, Italy), Professor E Fulcheri (Department of Pathology, University of Genoa, Genoa, Italy), Professor P Ghiorzo (Department of Internal Medicine and Medical Specialties [DiMI], University of Genoa, Genoa, Italy).

Financial \& competing interests disclosure

The authors have no relevant affliations or financial involvement with any organization or entity with a financial interest in or financial conflict with the subject matter or materials discussed in the manuscript. This includes employment, consultancies, honoraria, stock ownership or options, expert testimony, grants or patents received or pending, or royalties.

No writing assistance was utilized in the production of this manuscript.

Informed consent disclosure

The authors state that they have obtained verbal and written informed consent from the patients for the inclusion of their medical and treatment history within this case report.

\section{EXECUTIVE SUMMARY}

\section{Skeletal signs in Gorlin syndrome}

- Gorlin-Goltz or nevoid basal cell carcinoma syndrome is an autosomic dominantly inherited disorder associated to PTCH1 germline mutations recognized by a pool of clinical and radiologic signs. Since Kimonis et al. described major and minor criteria, other diagnostic criteria have been proposed and vary by source; however, few studies have tried to define the sensitivity and specificity of which phenotypic combination is most accurate for diagnosis.

\section{Sphenoid asymmetry in Gorlin syndrome}

- Abnormalities of the sphenoid bone are not very common, and consist of differently aggressive entities: some of them are typical of the pediatric age in few hereditary and congenital disorders.

- In the family described in this article, the helical and volume CT scan showed asymmetry of both sphenoid wings with thickening of the left wing, together with irregularity of the architecture of the trabecular bone. The trabecular bone showed alternating osteolytic and sclerotic areas; extensive multilamellar calcifications in falx cerebri and tentorium cerebelli were also present.

\section{Conclusion}

- Nevoid basal cell carcinoma syndrome diagnostic criteria should, therefore, be considered as dynamic entities and should be widened for the earlier and most accurate diagnosis of syndromic settings, especially after the widespread of novel and extremely accurate diagnostic techniques, such as volume and 3D CT scan. The inclusion of bifid ribs as a novel major criteria and the recognition of peculiar cranial anomalies such as sphenoid asymmetry, well detected at volume CT reconstruction, mayt be useful for the recognition and characterization of misdiagnosed cases.

\section{References}

1 Ponti G, Pollio A, Pastorino L et al. Patched homolog 1 gene mutation (p.G1093R9) induces nevoid basal cell carcinoma syndrome and non-syndromic keratocystic odontogenic tumors: a case report. Oncol. Lett. 4(2), 2412-2444 (2012)

2 Gorlin RJ, Goltz RW. Multiple nevoid basal-cell epithelioma, jaw cysts and bifid rib. A syndrome. N. Engl. J. Med. 262, 9089-9112 (1960).

3 Gorlin RJ, Sedano HO. The multiple nevoid basal cell carcinoma syndrome revisited. Birth Defects Orig. Artic. Ser. 7(8), 1401-1448 (1971).

4 Kimonis VE, Goldstein AM, Pastakia B et al. Clinical manifestations in 105 persons with nevoid basal cell carcinoma syndrome.
Am. J. Med. Genet. 69(3), 2993-3008 (1997).

5 Kimonis VE, Mehta SG, Digiovanna JJ et al. Radiological features in 82 patients with nevoid basal cell carcinoma (NBCC or Gorlin) syndrome. Genet. Med. 6(6), 4955-4902 (2004)

6 Satinoff MI, Wells C. Multiple basal cell naevus syndrome in ancient Egypt. Med. Hist. 13(3), 294-297 (1969).

7 Farndon PA, Del Mastro RG, Evans DG et al. Location of gene for Gorlin syndrome. Lancet 339 (8793), 581-582 (1992).

8 Pastorino L, Cusano R, Nasti S et al. Molecular characterization of Italian nevoid basal cell carcinoma syndrome patients. Hum. Mutat. 25(3), 322-323 (2005).
9 Derbent M, Bikmaz YE, Agildere M. A patient with Keipert syndrome and isolated fibrous dysplasia of the sphenoid sinus. Am. J. Med. Genet. A 155A(6), 149-169 (2011).

10 Ly JQ, Beall DP. Gorlin's syndrome: diffuse appendicular skeletal involvement with scintigraphic correlation. Australas. Radiol. 47(3), 3182-3181 (2003).

11 Ponti G, Pastorino L, Pollio A et al. Ameloblastoma: a neglected criterion for nevoid basal cell carcinoma (Gorlin) syndrome. Fam. Cancer 11(3), 411-418 (2012).

12 Keskin M, Karabekmez FE, Ozkan BT et al. Simultaneous occurrence of facial fibrous dysplasia and ameloblastoma. J. Craniomaxillofac. Surg. 37(2), 102-105 (2009). 
13 Iwanaga S, Shimoura H, Shimizu M et al. Gorlin syndrome: unusual manifestations in the sella turcica and the sphenoidal sinus. AJNR Am. J. Neuroradiol. 19(5), 956-958 (1998).

14 Lo Muzio L. Nevoid basal cell carcinoma syndrome (Gorlin syndrome). Orphanet. J. Rare Dis. 3, 32 (2008).

15 Lambrecht JT, Stubinger S, Siewert B et al. [Calcification of the falx cerebri. A pathognomonic symptom of Gorlin-Goltz syndrome]. HNO 53(8), 7014-7016 (2005).
Wattanasirichaigoon D, Prasad C, Schneider G et al. Rib defects in patterns of multiple malformations: a retrospective review and phenotypic analysis of 47 cases. Am. J. Med. Genet. Part A 122A(1), 63-69 (2003).

17 Shanley S, Ratcliffe J, Hockey A et al. Nevoid basal cell carcinoma syndrome: review of 118 affected individuals. Am. J. Med. Genet. 50(3), 2822-2890 (1994).

18 Bree AF, Shah MR; BCNS Colloquium Group. Consensus statement from the first international colloquium on basal cell nevus syndrome (BCNS). Am. J. Med. Genet. A. 155A(9), 20912-21097 (2011).

19 Sartip K, Kaplan A, Obeid G, Kadom N. Neuroimaging of nevoid basal cell carcinoma syndrome (NBCCS) in children. Pediatr. Radiol. 43(5), 6206-6227 (2013).

20 Lam C, Ou JC, Billingsley EM. 'PTCH'-ing it together: a basal cell nevus syndrome review. Dermatol. Surg. 39(11), 1557-1572 (2013). 